\title{
Processing of Visual Experimental PIV-Data Using a Random Synthetic Particle Generator
}

\author{
A.A. Mochalov',A,B, A. Yu. Varaksin²,A,B \\ A Moscow Bauman State Technical University, Russia \\ B Joint Institute for High Temperature, Russia \\ 1 ORCID: 00oo-0003-3078-1277, artem.mochalov@yandex.ru \\ 2 ORCID: 00oo-0o02-8799-6378, varaksin_a@mail.ru
}

\begin{abstract}
The study of free non-stationary vortex structures by the PIV method requires the search for the most rational approach to visual data processing. The paper evaluates the qualitative and quantitative characteristics of the velocity fields based on the generator of random synthetic particles. The simplified case of a solid-state rotation with a given velocity is considered as the closest to the vortex motion.

Keywords: generator of random synthetic particles, PIV, vector maps, numerical particle concentration.
\end{abstract}

\section{Introduction}

The fluid mechanics of single-phase flows uses a number of various methods of flow diagnostics including single point methods [1-3] and a wide class of contactless ones as well [4-13]. The latter, in turn, is divided into single point methods, first of all optical ones (e.g., LDA - laser Doppler anemometer [4-7]) and various modifications of the so called "field methods" [8-14in particular based on stroboscopic tracer visualization of flows. The latter allows obtaining of the instantaneous flow pattern what is of principal importance for the visualization and diagnostics of non-stationary turbulent flows [15-17] or that ones, containing large-scale vortex structures [18-22].

To measure the kinematic parameters of the gas medium using optical methods, tracer particles (of submicron and micron size) are introduced into it, and their mass and volumetric concentration is negligible. If the condition of the smallness of the relaxation time of the particles is observed in comparison with the characteristic scales of the carrier medium, the instantaneous velocities of the tracer particles will be almost equal to the velocities of the gas [23].

The particle image anemometry method is based on determining the displacements of tracer particles added to the flow and illuminated by a laser "knife". This method has an internationally accepted name PIV (Particle Image Velocimetry). The light source is usually a pulsed laser. In the experiment, a cross-correlation chamber is mounted perpendicularly to the laser knife direction and generates two images after a short period of time for the subsequent determination of the velocity field. For aerodynamical tasks, mist or microdrops of glycerin are the most commonly used trace particles.

This paper considers the processing of experimental photos based upon the standard cross-correlation algorithm the main idea of which consists in the following. The area of measurements is split into a number of elementary cells in which the average displacement of tracer particle images is calculated [24]. The parameter of the particles images density $\left(\mathrm{N}_{\mathrm{i}}\right)$ on experimental frames is of great importance for data processing by PIV algorithms due to its essential influence upon the accuracy of the instantaneous fields of velocities. The optimal density of particle images in a cell under calculation can significantly reduce the 
measurement error, since a larger number of trace particle pairs increases the clarity of the correlation peak [11]. In experimental images of the non-stationary vortex structure the density of particles images can vary within a wide range.

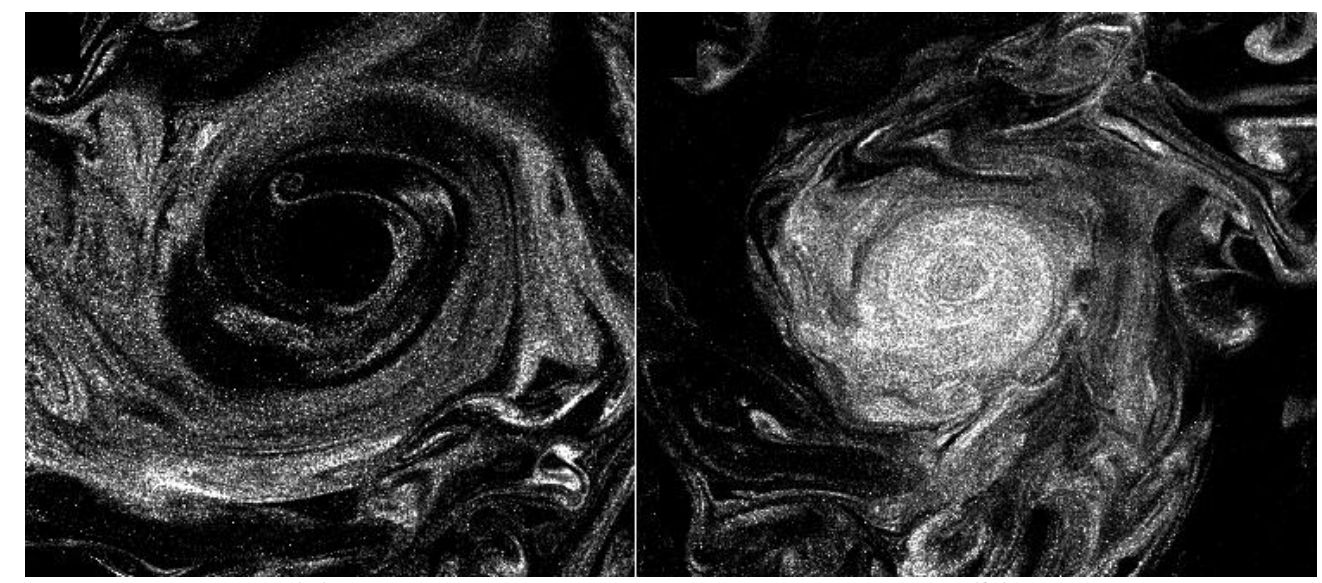

(a)

(b)

Fig. 1 An example of the real distribution of the particle concentration in the vortex core

In Fig. 1 two characteristic examples of the view of a non-stationary vortex structure are shown: (a) almost total absence of particles $\left(N_{i} \sim 0\right)$ and (b) - increased concentration of particles $\left(N_{i}>10\right)$. Further, based on the artificial particles generator, various approaches to the experimental frames processing are considered.

\section{Generator of the artificial trace particles}

It is possible to estimate the possible contribution of different densities of tracer particle images to the definition of vector maps using a software generator of artificial particles [25]. This software generates an image with a black background at a given resolution, onto which groups of white pixels are randomly applied with the aim of imitation of real particles in a flow. As initial established data for particles the following parameters can be distinguished: characteristic diameter and the deviation of its averaged value, the countable number of artificial particles in the frame, velocity of displacement etc. The main idea of using this software consists in obtaining of particle images with a given displacement for further processing with the use of PIV algorithms.

In order to find rational parameters for processing experimental images of vortex structures, various cases of seeding the flow with tracer particles were simulated. In Fig. 2 a collage of images with a different countable number of artificial particles is shown. Ones having characteristic diameter of 3 pixels are given as tracer particles. The displacement across the "knife" is not taken into account; the brightness of tracers is constant (Fig. 2).

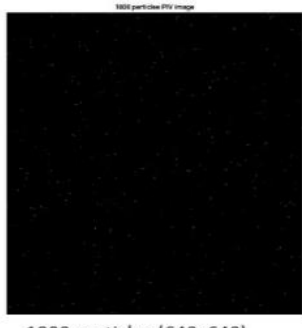

1000 particles $(640 \times 640)$

(a)

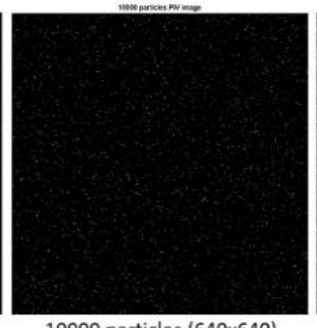

(b)

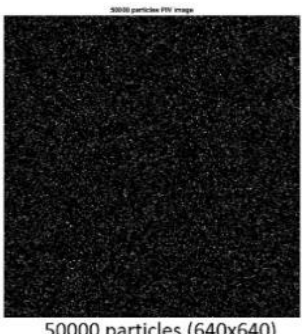

(c)

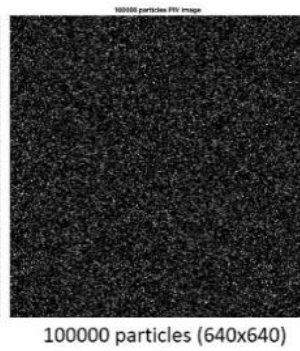

(d)

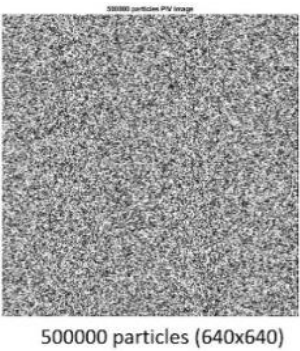

(e)

Fig. 2. Generated images of particles (artificial frame 1000x100o pixels): (a) - 103 particles; (b) $-10^{4}$ particles; (c) $-5 \cdot 10^{4}$ particles; (d) $-10^{5}$ particles; (e) $-5 \cdot 10^{5}$ particles 
The simulation of particles displacements is implemented for the case of a flat solid-state rotation; the distribution of velocities is linear, from the center to peripheral areas (Fig. 3).

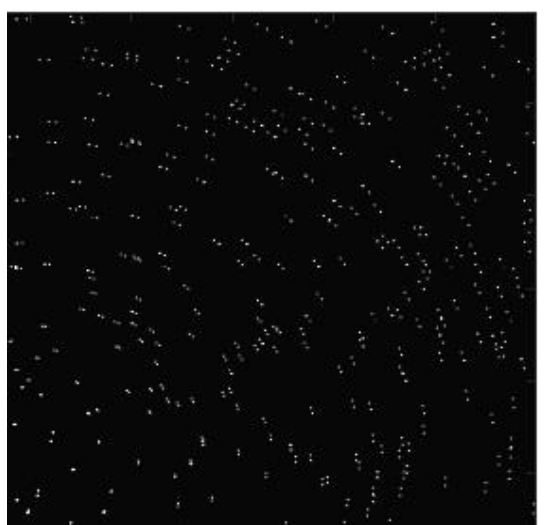

(a)

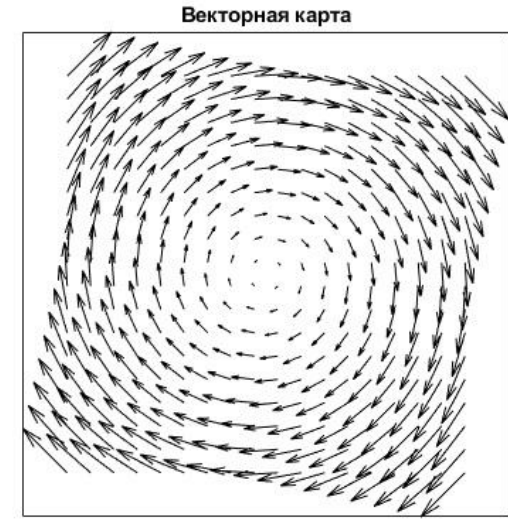

(b)

Fig. 3 Initial image of particles (a) and the vector map of a given turn

In Fig. 3 a segment (1/4) of particles image is shown, for clarity of rotation, the images in the cross-pair are superimposed on each other. Then, the optimal division into calculated cells is selected for the resulting image. As it is known [11], the size of a calculated cell affects the spatial resolution of the method. On one hand, the low number of initial calculated cells causes the low number of vectors. On the other hand, large number of cells results in the losses of data It should be noted that the well-known method of overlapping calculated cells equal to $50 \%$, although leads to a larger number of vectors, but does not qualitatively affect the spatial resolution of the method. In this paper we consider the instance of basic splitting of a pair of frames into calculated areas without overlapping areas. In Fig. 4 vector maps with different splitting of the original image into calculated areas are shown. The most typical partitions of the calculated grids into cells were selected, such as: $32 \times 32,64 \times 64,128 \times 128$ pixels. The number of the given grid elements can directly affect the quality and amount of the information about the object of investigations.

\section{Building of the vector maps for the twisted cross-section}

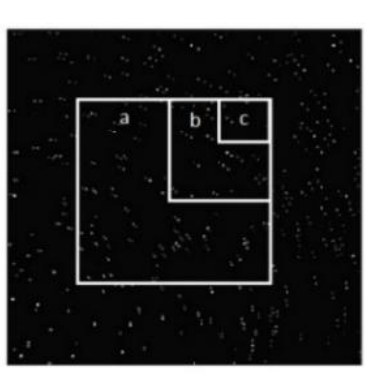

(a)

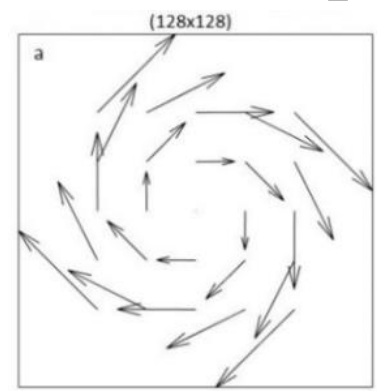

(b)

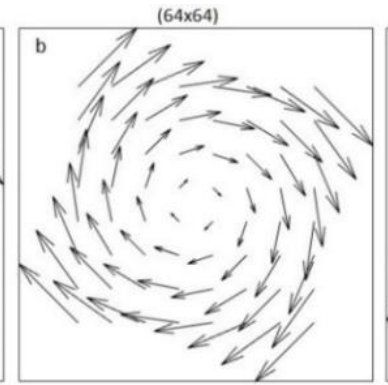

(c)

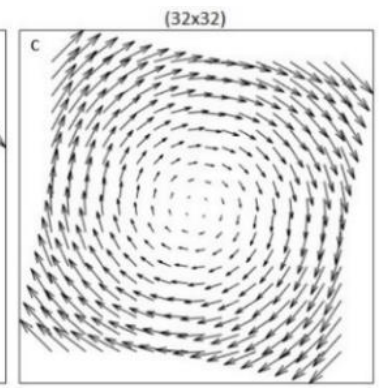

(d)

Fig. 4. Number of given vectors at various dimensions of the calculated area (artificial frame 1000x1000 pixels): (a) - artificial frame; (b) - 128x128 pixels; (c) - 64x64 pixels;

(d) $-32 \times 32$ pixels

In Fig. 5 the selected results of the PIV-algorithm application to artificial images with the fixed concentration of particles are shown. For clarity, examples of low concentration are given and the effect of vector loss is shown. 


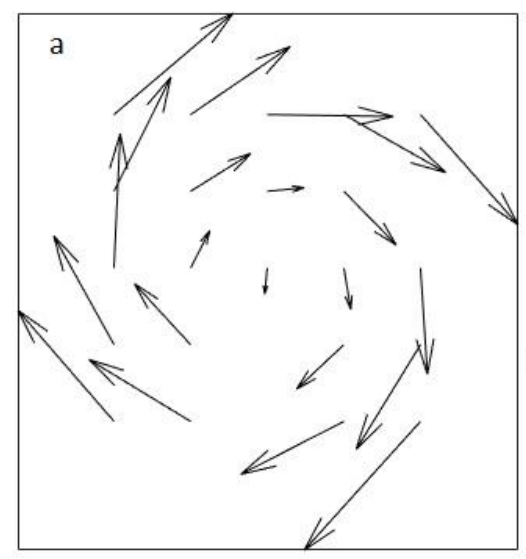

(a)

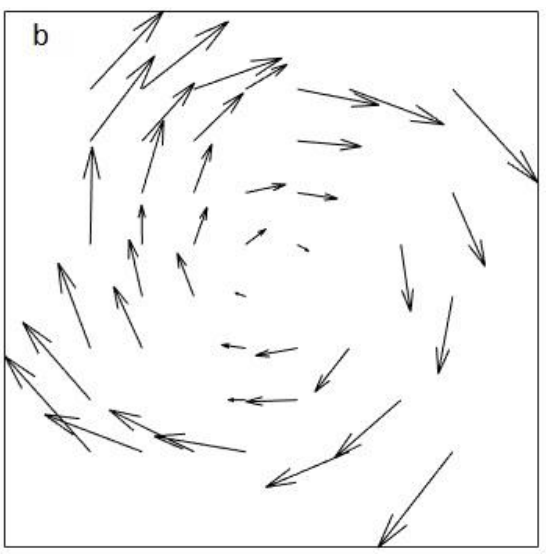

(b)

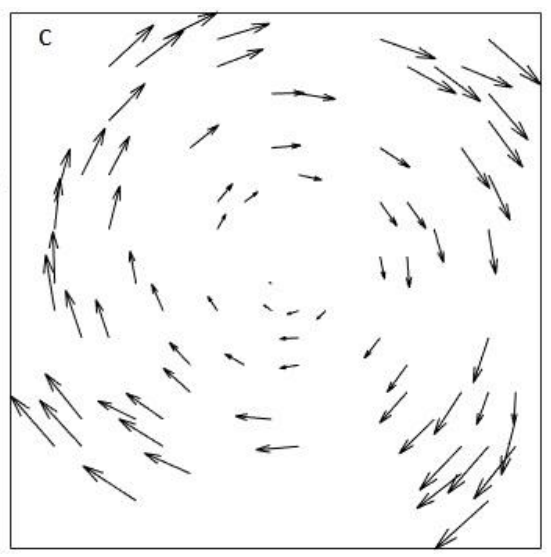

(c)

Fig. 5. Using of the cross-correlation algorithm of image processing at a low concentration of particles: (a) - 128x128 pixels; (b) - 64x64 pixels; (c) - 32x32 pixels

A summary graph of the number of vectors found depending on the concentration of particles in the frame is shown in Fig. 6.

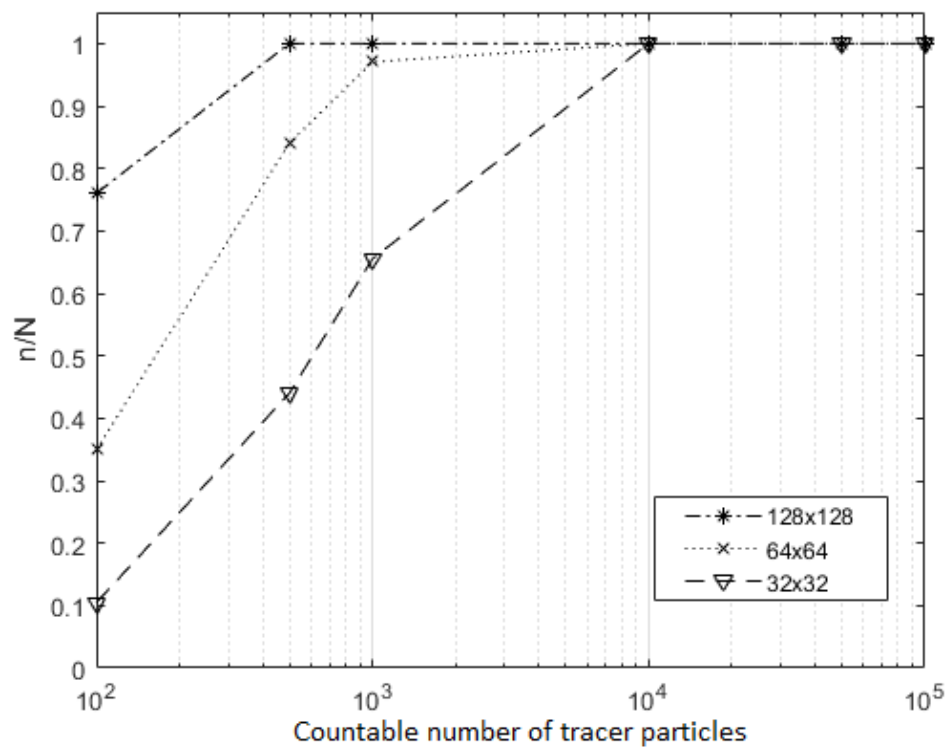

Fig. 6. Number of vectors determined

Fig. 6 shows that for low seeding by tracer particles splitting into large calculated areas is optimal (e.g., 128x128 pixels), but in this case the spatial resolution of the PIV method is lost. When decreasing of the calculated area and retaining of the low concentration of particles, the loss of vectors can reach $90 \%$ and $65 \%$ for $32 \times 32$ and $64 \times 64$ pixels, respectively. The optimal initial number of particles for vector maps determination (provided that good spatial resolution is maintained) are countable particles concentrations from $10^{4}$ (for frames of 1000x1000 pixels).

Among other things, the countable concentration of particles in the frame affects the quality of the vectors found. Here we consider the small dynamic range corresponding to the task of studying non-stationary concentrated vortices with low velocities (up to $10 \mathrm{~m} / \mathrm{s}$ ). An estimation of the deviation of vectors found from the initial ones obtained by the PIV method at various particles concentrations is shown in Fig. 7. 


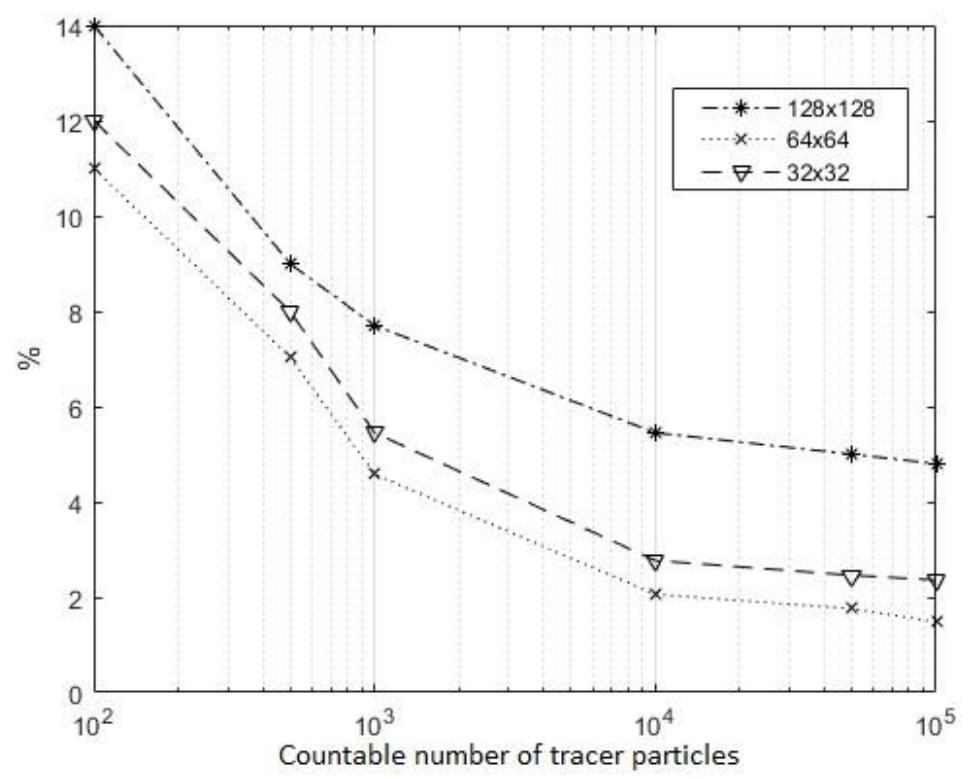

Fig. 7 Deviation of values of vectors found from the initial ones

The standard cross-correlation PIV algorithm follows from the assumption about the linear displacement of particles, and the deviation of the vectors found from the actual flow velocities is greater for essentially twisted flows. Within the framework of the task considered, the curvature of the current lines and the size of the calculated areas should be taken into account. At the optimal countable concentration of particles (104) and calculated cell size as well as the good spatial resolution the deviation can be of $2 \%$ and lower. Note that within the range of particles concentrations $10^{4}-10^{5}$ the quality of vectors determined does not change actually.

As an example, real fragments of the vortex structure with different concentrations of tracer particles are shown (Fig. 8a, 8b). Below are given fields of velocities with regular splitting into calculated areas of $32 \times 32$ pixels. In Fig. 8 (c) an example of vector loss caused by the insufficient seeding is shown, and inn Fig. 8 (d) the vector map is completed by the vectors with aid of the data interpolation.

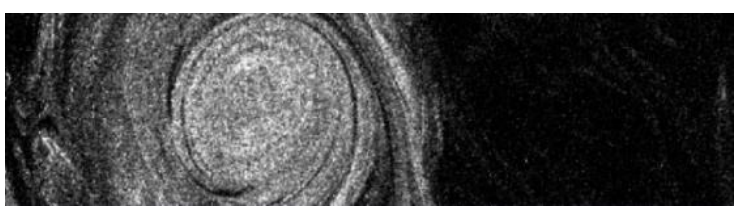

(a)

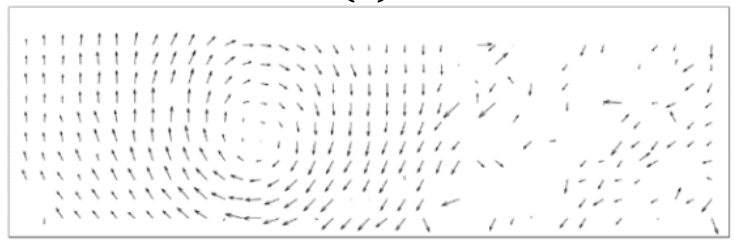

(c)

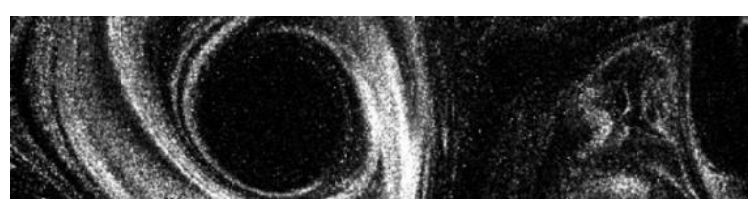

(b)

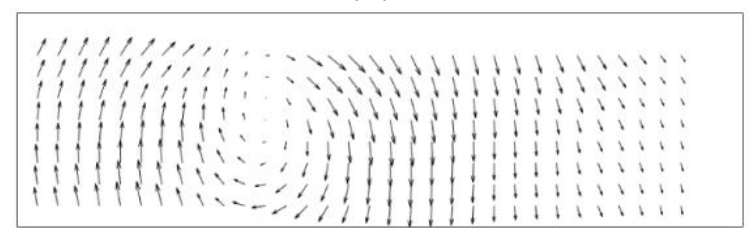

(d)

Fig. 8 Using of the fixed calculation area of application of a fixed computational domain $32 \times 32$ pixels; the images belong to one and the same series of frames for different moments of time: (a), (b) - images of the vortex structure; (c), (d) - fields of velocities.

It should be noted that for one vortex structure, the concentration of particles changes significantly at different times, for example, in the vortex funnel region, what leads to the loss of vectors. Using of the linear interpolation, in turn, allows completing a vector map by a number of vectors for the estimation of such parameters as the circulation and center of rotation. 


\section{Conclusion}

This paper shows various examples of experimental photos processing, provided that the resolution of the images is preserved. Estimates of measurement errors and number of the vectors lost at various splitting into calculated areas and different countable concentrations of tracer particles are given. The results of determination of real vortex structures are also given. The results obtained may be of interest for the tasks of processing of experimental photos of twisted flows with variable concentrations of tracer particles. Using of the generator of artificial particles, in the opinion of the authors, enables the simplification of the determination of the calculated cell size in order to obtain the maximum amount of information about the object under investigation in conditions of complex seeding by particles tracers in a real experiment.

\section{Acknowledgements}

The work is supported by the Russian Scientific Foundation (Project № 20-19-00551).

\section{References}

1. Bradshaw P. Turbulence. Springer - Verlag, Berlin, 1976. 338 p.

2. Yarin L.P., Genkin A.L., Kukes V.I. Termoanemometriya gazovyh potokov. L.: Mashinostroenie, 1983. $198 \mathrm{s.}$

3. Bruun H.H. Hot-Wire Anemometry: Principles and Signal Analysis. Oxford: University Press, 1995. 507 p.

4. Durst F., Melling A., Whitelaw J.H. Principles and Practice of Laser-Doppler Anemometry. London: Academic Press, 1976.

5. Durrani T., Greated C.A. Laser Systems in Flow Measurement. Springer, USA, 1977. 289 p.

6. Somerscales E.F.C. Laser Doppler Velocimeter. In Methods of Experimental Physics (ed. by Emrich R.J.). London: Academic Press, 1981. V.18 (fluid dynamics, part A). P. 93-240.

7. Dubnishchev Yu.N., Rinkevichyus B.S. Metody lazernoj doplerovskoj anemometrii. M.: Nauka, 1982. 303 s.

8. Adrian R.J. Particle-Imaging Techniques for Experimental Fluid Mechanics. Ann. Rev. Fluid Mech, 1991, vol. 23, p. 261-304.

9. Adrian R.J. Bibliography of Particle Velocimetry Using Imaging Methods: 1917-1995. TSI Inc., Minnesota, 1996.

10. Raffel M., Willert C., Kompenhans J. Particle Image Velocimetry. A Practical Guide. Berlin. Heidelberg: Springer-Verlag, 1998.

11. Raffel M., Willert C.E., Wereley S.T., Kompenhans J. Particle Image Velocimetry. Springer, 2007.

12. Westerweel J. Digital Particle Image Velocimetry - Theory and Application / Delft: Delft University Press, 1993. 235 p.

13. Westerweel J. Fundamentals of Digital Particle Image Velocimetry. Meas. Sci. Technol, 1997, vol. 8, p. 1379-1392.

14. Bilsky A.V., Gobyzov O.A., Markovich D.M. Evolution and Recent Trends of Particle Image Velocimetry for an Aerodynamic Experiment (Review). Thermophysics and Aeromechanics, 2020, vol. 27, p. 1-22.

15. Varaksin A.Yu. Collision of Particles and Droplets in Turbulent Two-Phase Flows. High Temperature, 2019, vol. 57, p. 555-572.

16. Varaksin A.Yu. Two-Phase Boundary Layer of Gas with Solid Particles. High Temperature, 2020, vol. 58, p. 716-732.

17. Okulov V.L., Naumov I.V., Kabardin I.K., Litvinov I.V., Markovich D.M., Mikkelsen R.F., Sorensen J.N., Alekseenko S.V., Wood D.H. Experiments on Line Arrays of HorizontalAxis Hydroturbines. Renewable Energy, 2020, vol. 163, p. 15-21. 
18. Dulin V.M., Lobasov A.S., Chikishev L.M., Markovich D.M., Hanjalic K. On Impact of Helical Structures on Stabilization of Swirling Flames with Vortex Breakdown. Flow Turbulence and Combustion, 2019, vol. 103, p. 887-911.

19. Lobasov A.S., Alekseenko S.V., Markovich D.M., Dulin V.M. Mass and Momentum Transport in the Near Field of Swirling Turbulent Jets. Effect of Swirl Rate. International Journal of Heat and Fluid Flow, 2020, vol. 83, paper 108539.

20. Sharaborin D.K., Tolstoguzov R.V., Dulin V.M., Markovich D.M. On the Structure of an Impact Jet with Flow Swirling and Combustion. Combustion Explosion and Shock Waves, 2020, vol. 56, p. 131-136.

21. Varaksin A.Yu., Mochalov A.A. A Double Screw Spiral as a Possible Mechanism of Wall-Free Nonstationary Air Vortex Generation. Doklady Physics, 2019, vol. 64, p. 301-303.

22. Varaksin A.Yu., Denshchikov K.K., Protasov M.V., Romash M.E. Visualization of Whirlwind (Non-Stationary Vortex) Structures Aimed to the Improvement of Cooling Systems of Electric Power Devices, Scientific Visualization, 2020, vol. 12, p. 74-83.

23. Varaksin A.Yu. Two-Phase Flows with Solid Particles, Droplets, and Bubbles: Problems and Research Results (Review). High Temperature, 2020, vol.58, p. 595-614.

24. Raffel M., Leitl B., Kompenhaus J. Data validation for particle image velocimetry. Laser Techniques and Application in Fluid Mechanics, Springer - Verlag, 1993, pp. 210 - 216

25. Thielicke W., Stamhuis E.J. PIVlab - Towards User-friendly, Affordable and Accurate Digital Particle Image Velocimetry in MATLAB. Journal of Open Research Software, 2014, vol. 2, p.e30, DOI: http://dx.doi.org/10.5334/jors.bl 